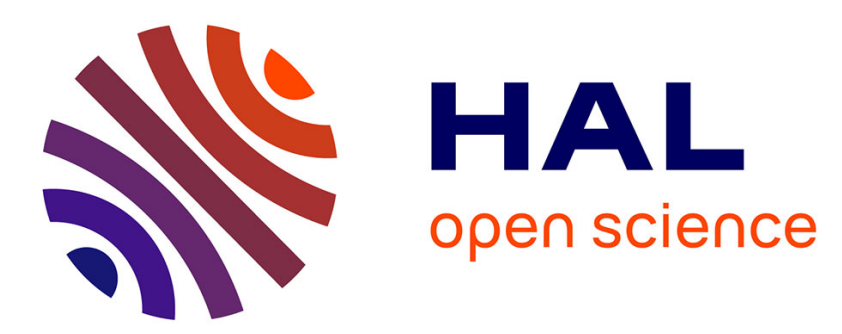

\title{
Quelques remarques sur les bullæ inscrites de la ville basse de Kültepe
}

\author{
Cécile Michel
}

\section{To cite this version:}

Cécile Michel. Quelques remarques sur les bullæ inscrites de la ville basse de Kültepe. Bérengère Perello, Aline Tenu. Parcours d'Orient Recueil de textes offert à Christine KEPINSKI , p. 175-184, 2016. halshs-01674035

\section{HAL Id: halshs-01674035 https://shs.hal.science/halshs-01674035}

Submitted on 4 Jan 2018

HAL is a multi-disciplinary open access archive for the deposit and dissemination of scientific research documents, whether they are published or not. The documents may come from teaching and research institutions in France or abroad, or from public or private research centers.
L'archive ouverte pluridisciplinaire HAL, est destinée au dépôt et à la diffusion de documents scientifiques de niveau recherche, publiés ou non, émanant des établissements d'enseignement et de recherche français ou étrangers, des laboratoires publics ou privés. 


\title{
PARCOURS D'ORIENT RECUEIL DE TEXTES OFFERT À CHRISTINE KEPINSKI
}

\author{
sous la direction de \\ Bérengère Perello et Aline Tenu
}




\section{ARCHAEOPRESS PUBLISHING LTD \\ Gordon House \\ 276 Banbury Road \\ Oxford OX2 7ED}

www.archaeopress.com

ISBN 9781784914585

ISBN 9781784914592 (e-Pdf)

(C) Archaeopress and the authors 2016

All rights reserved. No part of this book may be reproduced, in any form or by any means, electronic, mechanical, photocopying or otherwise, without the prior written permission of the copyright owners.

Printed in England by Oxuniprint, Oxford

This book is available direct from Archaeopress or from our website www.archaeopress.com 


\section{Table des matières}

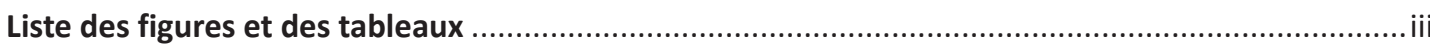

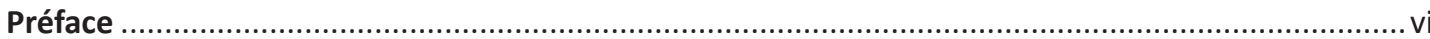

Bibliographie de Christine Kepinski...................................................................................... vii

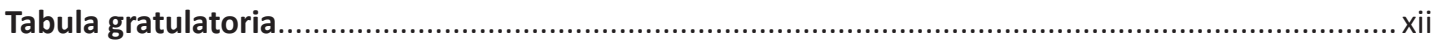

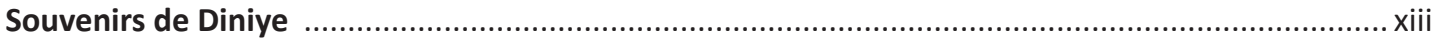

Hélène David-Cuny \& Joël Suire

Étude croisée sur un plan d’urbanisme irrégulier du Bronze ancien : le cas de Titriş Höyük 1

Christophe Benech

A Sealing from Tell Arbid: once more about seal impressions on ceramic vessels 9 Piotr Bieliński

La période de Halaf à Kutan, Irak

Catherine Breniquet

Internal-Handled Bowls - Puzzling pots from Bronze Age Mesopotamia

Ulrike Bürger \& Peter A. Miglus

L'Hinterland mariote en question : quelques réflexions

Pascal Butterlin

Des outils agricoles en pierre du Bronze ancien de Syrie : les têtes d'araires

Corinne Castel

La « maison de Riš-Šamaš » à Harradum, nouvelle approche.

Dominique Charpin

Un sceau-cylindre mitannien de Tell Afis (Syrie).

Barbara Chiti

La chronologie politique du Suhu au VIII ${ }^{\mathrm{e}}$ siècle

Philippe Clancier

Inscriptions syriaques de Tilbeşar

Alain Desreumaux

Affaires de Famille?

Jean-Marie Durand

The Middle Euphrates, Iraq: Assyrian-Babylonian interactions in an Aramaean territory in the early 1st millennium BC

Arnulf Hausleiter 
À propos des pommeaux de chars

Jean-Louis Huot

By the streets of Babylon

Francis Joannès

The Sheikh Hamad / Dūr-Katlimmu Texts DeZ 2521 and DeZ 3293 and their Implications for a Middle Assyrian Supra-Regional Canal

Hartmut Kühne

Que se passait-il à Kunara il y a quatre mille ans... ?

Bertrand Lafont

5500 av. notre ère : le vase de Marawah MR11 et l'Obeid du Golfe

Sophie Méry, Michael James Blackman, Mark Jonathan Beech et Kevin Lidour

Quelques remarques sur les bullæ inscrites de la ville basse de Kültepe

Cécile Michel

Note sur quelques sites anciens dans la région de Samarra

Alastair Northedge

L'émergence du phénomène urbain en Anatolie : état de la question

Bérengère Perello

Les briques inscrites de Qasr Shemamok migrations, réutilisations et valeur documentaire.... 209 Olivier Rouault et Maria Grazia Masetti-Rouault*

La forteresse médiévale de Tilbeshar (Tell Bashir, Turbessel)

Marie-Odile Rousset

Le moyen Euphrate dans l'iconographie néo-assyrienne

Aline Tenu 


\title{
Quelques remarques sur les bullæ inscrites de la ville basse de Kültepe
}

\author{
Cécile Michel*
}

\begin{abstract}
Résumé
Selon les sources écrites exhumées à Kültepe (Anatolie centrale) et datant du XIXe siècle avant J.-C., l'usage des bullae était répandu. Il s'agit de morceaux d'argile appliqués sur un contenant, une porte ou un ballot de marchandises, et qui comportent souvent une ou plusieurs empreintes de sceaux, et parfois un petit texte en cunéiforme. Toutefois, en cinquante années de fouilles, les archéologues en ont découvert un nombre limité dans les maisons privées de marchands de la ville basse. En fonction de leur forme et du texte écrit sur certaines d'entre elles, il convient de restituer à chaque bullæ son usage dans l'Antiquité. Malheureusement, pour celles ayant servi à la classification des archives, elles ont été inventoriées à part, séparément des tablettes. À partir de l'étude de lots d'archives archéologiques cohérents, les assyriologues tentent donc de reconstituer le puzzle en découvrant quelles tablettes correspondent à une bullæ donnée.
\end{abstract}

Mots-clés : Kültepe, Kaneš, bullæ, scellement, paléo-assyrien, classement des archives

\begin{abstract}
According to written sources from Kültepe (Central Anatolia), the use of bullæ was widespread. This word refers to lumps of clay applied to a container, a door or a bundle of merchandise, which often present one or more sealings, and sometimes a short cuneiform text. However, there are surprisingly not so many bullæ found in the private houses of merchants located in the lower town during fifty years of excavations. With the help of their shape and of the text written on some of them, we have to guess the use of each bullæ in the 19th century BC. Unfortunately, for the bullæ originally used to file archives, they were inventoried separately from tablets. From the study of coherent archaeological archives, Assyriologists try to put together the puzzle by searching which tablets correspond to a given bullæ.
\end{abstract}

Keywords: Kültepe, Kaniš, bullæ, sealings, Old Assyrian, filing archives

Exploré en continu depuis plus de soixante ans, le site de Kültepe, l'ancienne Kaneš, situé non loin de Kayseri en Anatolie centrale, figure parmi les sites du ProcheOrient les plus prolixes en tablettes cunéiformes. En effet, près de 22500 tablettes y ont été exhumées - dont 17700 découvertes lors des fouilles régulières entamées en 1948 - provenant pour l'essentiel de maisons de marchands assyriens installés dans la ville basse ; seules 40 tablettes furent trouvées sur la citadelle ${ }^{1}$. En comparaison, le nombre de scellements, désignés sous le terme bullæ par les spécialistes de Kültepe ${ }^{2}$, paraît très faible. Entre 1948 et 2005, les archéologues n’ont

\footnotetext{
CNRS, Archéologies et Sciences de l'Antiquité, Nanterre ; cecile. michel@mae.cnrs.fr. Il m'est particulièrement agréable d'offrir cette étude à Christine Kepinski qui, outre la direction de quatre chantiers de fouilles en Irak (Khirbet ed-Diniyeh, Tell Khoshi, Grai Resh, Kunara), a dirigé pendant presque quinze ans la mission archéologique de Tilbeshar à l'est de l'Anatolie. J'adresse tous mes remerciements à F. Kulakoğlu qui m'autorise à reproduire les photos du matériel de la mission qu'il dirige.

1 Michel $2003 ; 2006 ; 2011$ a ou b.

2 Un bullce est un morceau d'argile appliqué sur un contenant, une porte ou un ballot de marchandise, et qui comporte souvent une ou plusieurs empreintes de sceaux, et parfois un petit texte en cunéiforme. La terminologie pour désigner les scellements (sealings, en anglais) n'est pas fixe. À Arslantepe, les archéologues préfèrent le mot cretulce, cf. Frangipane et al. 2007. Notons que la notion de scellement n'est pas suffisamment précise si ce mot renvoi à l'action de sceller ou au résultat de cette action. En effet, le scellement de bullæ ne reflète qu'une des pratiques du scellement à Kültepe, la plupart des empreintes de sceaux recensées figurant sur des enveloppes de tablettes.
}

mis au jour que 430 bullæ à Kültepe, dont 419 sont publiées $^{3}$. Un peu plus d'un quart (118) de ces bullæ ont été découvertes sur la citadelle. N. Özgüç a comparé ces données à celles d'Acemhöyük, au sud du Tuz Gölü (Lac Salé), qui a livré environ 1300 bullæ trouvées surtout dans deux pièces de stockage $(6,42)$ du bâtiment public appelé Sarıkaya, contemporain des archives de Kültepe ${ }^{4}$. L'absence de bullæ dans les palais de Kültepe des niveaux 8 et 7 (contemporains des niveaux II et Ib de la ville basse qui ont livré des tablettes) s'explique par le très mauvais état de ces ruines, endommagées par les explorations antérieures à 1948. Seuls 4 bullæ ont été mises au jour dans le palais de la terrasse sud (niveau 8), et 31 dans le palais de Waršama (niveau 7), dont une de forme triangulaire et inscrite $(\mathrm{Kt} \mathrm{z} / \mathrm{t} 15)^{5}$. Or depuis 2010, les archéologues travaillent, sur la citadelle de Kültepe, au dégagement d'une structure monumentale d'au moins 70 x 55 m, qui daterait de la fin du Bronze ancien (niveau $13)^{6}$. Dans la partie sud-ouest de ce bâtiment, une immense fosse a livré, d'ores et déjà, près de deux milles fragments de bullæ, portant souvent des empreintes de sceaux tampons ou sceaux-cylindres sur une face, et des traces de fil, de corde, de textile, de panier, de bois, etc.

\footnotetext{
3 Özgüç N. et Tunca 2001.

4 Özgüç N. In Özgüç N. et Tunca $2001: 127$.

Larsen $2008: 83$.

${ }^{6}$ Ezer 2014 ; Kulakoğlu 2015.
} 
sur l'autre face ${ }^{7}$. Le nombre très élevé de bullæ provenant de ce complexe monumental témoigne de la gestion administrative d'un grand ensemble de la fin du Bronze ancien (BA III). Cette situation n'est pas sans rappeler les découvertes de Karahöyük-Konya, où de nombreux scellements ont été découverts dans une fosse profonde (niveau I, tranchée L) ${ }^{8}$.

La présente étude porte sur les bullæ exhumées entre 1948 et 1997 dans les maisons privées de la ville basse, et qui ont fait l'objet d'une publication par N. Özgüç et Ö. Tunca (2001). Selon ces auteurs ${ }^{9}, 240$ bullæ ont été découvertes dans les « archives » des marchands, la plupart dans des pièces contenant également de la céramique fine, des objets en métal et divers autres artefacts. Le mot « archives » est sans doute à comprendre ici comme " pièce recelant les archives (et les marchandises) », toutes les bullæ n’étant pas nécessairement liées aux tablettes.

\section{La forme des bullæ}

N. Özgüç a recensé les différents usages de ces bullæ en fonction de leur forme ${ }^{10}$ :

- bullae hémisphéroïdes qui, d'après les marques de ficelles et textiles au dos, ont servi à sceller des ballots en textile ou des petites céramiques,

- bulla triangulaires perforées pour le passage d'une ficelle afin de fermer un ballot ou un contenant,

- bullae en forme de pignon avec marques de ficelles et nœuds de ficelles sur leur envers pour clore des vases au col étroit,

- bullce en forme de cône avec un trou de ficelle sur le haut, et des impressions de sceaux tampons employés par les autochtones, attachées par une ficelle à des marchandises,

- bullce en forme de tablette avec impressions de sceaux-cylindre,

- bulla en forme de demi-anneau avec trou de ficelle et empreintes de sceaux (sous-groupe des hémisphéroïdes),

- bullae en forme de disque ou de plaque, utilisées comme couvercle (?),

- bullae servant de bouchons en forme de champignon, de disque ou pour fermer des pichets à bec verseur,

- bullce trouées avec empreintes de ficelles sur le bord du trou ; l'une devait être attachée à un récipient.

\footnotetext{
7 Cette fosse n'a pas encore été totalement vidée. Le tamisage systématique de la terre provenant de la fosse a permis de ramasser tous les fragments de bullce. Une étude systématique des traces laissées sur la face interne de ces bullae est en cours par E. Andersson Strand, C. Breniquet et C. Michel dans le cadre du programme international de collaboration scientifique Textiles de l'Orient à la Méditerranée, puis du groupement de recherche international Ancient Textiles from Orient to the Mediterranean (http://www.mae.u-paris10.fr/gdri-atom/).

8 Alp 1968 : 12-18.

9 Özgüç N. et Tunca $2001: 131$.

${ }^{10}$ Özgüç N. et Tunca $2001: 135-136$.
}

Il en ressort que ces bullce ont servi à sceller toutes sortes de contenants (ballots, caisses, paniers, céramiques) et certaines, comportant de profondes rainures sur leur envers ou des marques de ficelles sur une surface plutôt lisse auraient pu être utilisées pour sceller des portes ${ }^{11}$.

\section{Usages des bullæ selon les données textuelles}

Les textes témoignent d'un usage intensif de la pratique du scellement par les marchands, en dehors des sceaux apposés sur les enveloppes ${ }^{12}$. Tous les envois de marchandises depuis Aššur vers l'Anatolie, ou d'un lieu à un autre en Anatolie centrale : or, argent, étain, cuivre, textiles, pierres semi-précieuses, voire des denrées alimentaires ${ }^{13}$, se faisaient dans des emballages (textiles, sacs en cuir, paniers ou nattes de roseaux) ou contenants divers scellés à l'aide d'un morceau d'argile sur lequel était imprimé le sceau du propriétaire ou de l'expéditeur. De même, dans les maisons de la ville basse de Kaneš, les marchandises étaient stockées dans leurs contenants (tamalakkum, siliānum) sous scellés, euxmêmes entreposés dans des coffres, recoins et pièces scellées (maknakum, mașșartum) ${ }^{14}$. Lorsqu'un marchand décédait, sa maison était généralement placée sous scellés en attendant le règlement de ses créanciers, avant que la succession ne puisse avoir lieu ${ }^{15}$.

Dans les pièces et espaces scellés, les marchands conservaient aussi leurs archives, classées et rangées dans des sacs, caisses et céramiques, souvent euxmêmes scellés ${ }^{16}$. La garde des marchandises et des tablettes incombaient aux femmes qui demeuraient dans les maisons ${ }^{17}$ : «Entrez chez Nuhšatum et qu'elle place devant vous tous les récipients de tablettes certifiées de notre père. Brisez les scellements d'Aššur-rabi et que deux employés honnêtes les scellent de nouveau de leurs sceaux. Puis remettez-les au sein des récipients, scellez

\footnotetext{
11 Özgüç N. et Tunca 2001: 134.

${ }^{12}$ Larsen 1977, 2008 ; Veenhof 1993.

${ }^{13}$ BIN 4, 85 :4-13, « Mașī-(i)lī, le fréteur de Mannum-balum-Ana t'apporte une étoffe-kutānum de qualité ordinaire pour Šupiahšušar, un châle akkadien pour Umminara (et) un châle akkadien pour Hattītum sous mon sceau et sous le sceau d'Anini, ta fille » (Michel 2001 : n³71). TC 2, 47:4-10, « Šamaš-bāni t'apporte à la fois 55 mines de saindoux (dans) un sac en cuir sous mon sceau et le malt-narūtum qui m’est parvenu (Michel 2001 : n³58; Larsen 2002 : n52).

${ }^{14}$ BIN 6, 20:3-12, « Pourquoi m'as-tu écrit ainsi : “Je vais ouvrir la pièce scellée et je vendrai le pain de bière." Le rez-de-chaussée de même que l'étage, n'est-ce pas par mon sceau qu'ils sont scellés ? Je t'en prie, jusqu'à mon arrivée, n'ouvre aucun sceau sur la maison, te surveille attentivement mes sceaux (qu'ils restent intacts)! » (Michel 2001, n³34). VS 26, 53:5-18, «(Il y a) 8 talents 50 mines d'étain sous scellés dans le magasin de la pièce forte de devant, 230 étoffes ordinaires, 120 étoffes de bonne qualité (et) 13 ânes noirs, tout cela, je te l'ai laissé dans la maison sous scellés. » (Michel 2001, n³62). Kt 93/k 69:18-27, « Nous avons ouvert les contenants-tamalakkum en 1'air (sur des étagères ?) qui étaient emballés dans du cuir, et (...) en avons extrait la tablette ». ta-ma-lá-ki : e-li-[i], ša i-na ma-áš-ki-im, ha-ar-mu-ni : ni-ip-té-[ma] (...) tup-pá-am nu-šé-li-ma.

${ }^{15}$ Michel 1994 ; Veenhof 2011 ; Hertel 2013 : 337-380.

${ }^{16}$ AKT 3, 106 : 7-14, « Tu es ma mère. Le jour où tu entendras ma lettre, (prends) les caisses-tamalakkum de tablettes certifiées qu'Aššurrabi t’a laissées » (Michel $\left.2001: n^{\circ} 290\right)$.

${ }^{17}$ TPAK 1, 63, cf. Michel 2009.
} 
les tablettes et qu'elles demeurent chez Nuhšatum. » Ce passage documente la pratique du double scellement des tablettes par différentes personnes.

\section{Les données archéologiques concernant le contexte de découverte des bullæ}

La présence de bullce dans des pièces contenant à la fois des archives et des marchandises ne permet pas, en l'absence de rapport de fouilles, de connaître la relation qui pourrait exister entre les bullæ et les tablettes. Les descriptions du contenu de certaines maisons de la ville basse de Kütltepe donnent toutefois un peu plus de précisions.

Les archives d'Elamma, mises au jour en 1991 et 1992 et éditées par K. R. Veenhof ${ }^{18}$, ont été décrites par T. Özgüç comme suit ${ }^{19}$ : «The archive of the merchant was found along the base of the east wall of room 3 and in rooms $4-5$, in groups once packed in boxes, bags, sacks and straw mats. On top of each group lay one or two bullce. Unopened envelopes were placed at the bottom, tablets on top. In contrast to other archives, here we did not find tablets stored in jars. »

Les tablettes étaient donc classées par lots, emballées dans des contenants en matériaux souvent périssables dotés chacun d'une étiquette d'argile ou bulla qui en indiquait vraisemblablement le contenu. Malheureusement, lors du ramassage du matériel, bullce et tablettes ont été séparées et inventoriées à part ${ }^{20}$. Les assyriologues en charge d'archives à publier, provenant d'une maison donnée, tentent donc de reconstituer les lots originaux en fonction du contenu des textes et de rechercher dans les bulla celles ayant pu servir à identifier chacun des lots. En dehors des empreintes de sceaux, pour lesquelles un travail de grande ampleur est encore à mener, le bref texte présent sur quelques bullce permet de se faire une idée du contenu du récipient ou du ballot auquel elles étaient attachées. Cette recherche doit également prendre en compte la forme de la bulloe analysée, ainsi que les marques présentes sur son envers.

\section{Les bullæ inscrites}

Ö. Tunca a recensé 144 bullae inscrites et en propose une classification en cinq catégories et dix sous-groupes ${ }^{21}$. M. T. Larsen suggère de ne distinguer que deux types d'inscriptions ${ }^{22}$ :

- celles utilisant le mot našpertum qui définit l'envoi de marchandises ou de tablettes à caractère juridique ${ }^{23}$; et pour un même usage, celles portant simplement la mention « pour NP»,

\footnotetext{
${ }^{18}$ AKT 8.

${ }^{19}$ T. Özgüç $1994: 369$

${ }^{20}$ Veenhof $2013: 58$.

${ }^{21}$ Tunca, In N. Özgüç et Tunca 2001 : 305-307.

${ }^{22}$ Larsen 2008 : 83.

${ }^{23}$ Veenhof $1993: 648-649 ; 2003: 88-89$. Selon K.R Veenhof (Veenhof 2013 : 51), une bulla portant l'inscription "našpertum de NP »
}

- celles employées à des fins d'archivage, avec par exemple l'indication « tablettes... ».

Notons toutefois que ces deux catégories ne peuvent être exclusives ; en effet, certains des contenants utilisés pour le transport de biens ou de tablettes ont aussi pu servir à leur stockage, tout au moins temporaire.

Les publications d'archives découvertes en contexte archéologique sont encore peu nombreuses ; en revanche, un certain nombre sont en cours d'étude. En outre, certaines maisons n'ont pas livré de bulloe, ou seulement des bullæ non inscrites. Ainsi, la maison d'Ennum-Aššur, fils de Šalim-ahum, dont les archives ont été partiellement publiées en 1995 (AKT 3, Kt v/k) ne semble pas avoir contenu de bullae ${ }^{24}$. On peut noter aussi l'absence de bullce dans la maison de Peruwa, un Anatolien qui a laissé une archive exhumée en partie en $1951(\mathrm{Kt} \mathrm{d} / \mathrm{k})^{25}$. La maison de Galulu, un autre Anatolien, explorée en 1964 contenait deux bullae anépigraphes (Kt $\mathrm{f} / \mathrm{k})$.

Certaines identifications entre bulloe inscrites et tablettes semblent faciles à établir. Ainsi, en 1963 (Kt o/k), les archéologues ont mis au jour deux archives assyriennes totalisant 207 tablettes et enveloppes ${ }^{26}$. Ils ont aussi découvert 10 bullce - dont huit inscrites - provenant d'une maison de la ville basse (niveau II), située dans le carré B-D/11-12 27. L'une de ces bulloe portant le texte « tablettes concernant le testament d'Agūa » (Kt o/k 203) a pu être mise en relation avec le testament lui-même $(\mathrm{Kt} \mathrm{o} / \mathrm{k} 196 \mathrm{a}, \mathrm{c})^{28}$. D'autres tablettes liées à ce testament devaient être conservées dans le même contenant : titres de propriété des maisons d'Aššur et de Kaneš, créances, etc. ; elles n'ont pu être identifiées parmi les tablettes publiées. Cet exemplaire non ouvert du testament d'Agūa aurait pu être conservé dans un lot d'archives appartenant à Šū-Bēlum, fils du défunt. Il pourrait tout aussi bien s'agir d'une copie de témoin, conservée intacte dans les archives de ce dernier.

renverrait soit à une tablette certifiée, soit à un paquet contenant une enveloppe certifiée. Il ne s'agirait pas de marchandises ; il précise, p. 57, que le mot našpertum signifie « missive ».

${ }^{24}$ Les bullae exhumées en 1970 seraient toutes originaires du palais de Waršama, N. Özgüç et Tunca $2001: 182$.

${ }^{25}$ Michel 2011 b.

${ }^{26}$ Une partie de ces textes ont été publiés par İ. Albayrak (Albayrak 2006) dans le volume (A)KT 4, cf. le compte-rendu de l'ouvrage par Veenhof 2009. Beaucoup de textes trouvés cette année-là ont aussi fait l'objet de publications séparées Albayrak 1996 (Kt o/k 39, 40, 44, 46, 64, 81 et 106) ; Albayrak 2000 (Kt o/k 196c et de son enveloppe Kt o/k 196a); Albayrak 2001 (Kt o/k 52) ; Albayrak 2005a (60, 74, 75) ; Albayrak 2005b (45, 47, 49, 50, 53, 72) ; Albayrak 2006 (117, 134, 142, 163, 172, 187, 195) ; Erol et Albayrak $2012(\mathrm{Kt} \mathrm{o} / \mathrm{k} 68,122$, 149, 154, 157 et 160) ; Öz et Albayrak 2012 (70, 90, 120, 128, 146 et 147).

${ }^{27}$ Ces bullae proviennent de la pièce 3 de la maison, N. Özgüç et Tunca 2001: 174-177, 327-329. Nous ne savons a priori pas si l'une des deux archives est issue de la même pièce.

${ }^{28}$ Michel 2000. Il aurait été intéressant de pouvoir comparer les six sceaux apposés sur l'enveloppe du testament avec celui d'Apil-kēn, fils d'Ilī-dān, présent sur la bullce. 


\section{Les bullæ dans la maison de Šumī-abiya (1990)}

En 1990, les archéologues ont mis au jour deux archives dans les carrés LVII-LX/132-134. L'une de ces archives a été publiée : elle provient de la maison de Šumīabiya, fils de Puzur-Ištar, et comporte un lot de textes appartenant à Aššur-mūtappil, fils d'Iddin-abum ${ }^{29}$. Selon N. Özgüç, cette archive contenait également 17 bullae; seulement 7 sont décrites et 2 autres sont citées dans le commentaire ${ }^{30}$. Celles-ci, portant des marques de ficelles sur le revers, auraient servi à sceller une porte (Kt 90/k 48, Kt 90/k 111) ; la seconde possède une inscription qui mentionne le sceau de Țāb-Aššur et un envoi d'Aššur$\operatorname{id}^{31}$. Le texte va donc à l'encontre d'une identification à un scellement de porte ; si l'empreinte sur l'envers de la bulloe fait penser à scellement d'un objet en bois, ce dernier pourrait être un coffre contenant marchandises ou tablettes, envoyé depuis Aššur ou une autre localité. On peut éventuellement relier cette bulloe avec la lettre TPAK 1, 14 expédiée par Aššur-idī à l'attention d'Aššurmūtappil et Țāb-Aššur, même si cette dernière ne fait aucune allusion à un envoi. Trois autres bullce de ce lot sont inscrites. La bullce Kt 90/k 206, qui devait être attachée à un contenant renfermant des verdicts émis par le kārum de Kaneš concerne une affaire en justice impliquant Abu-šalim, fils de Kurara ; or ce dernier n'est pas attesté dans l'archive de Šumī-abiya ${ }^{32}$. La bullce Kt 90/k 209 porte en son revers des rainures fines et parallèles. Un texte fragmentaire mentionnant des individus et leurs patronymes est inscrit, mais seuls les noms des patronymes subsistent ${ }^{33}$. La bulloe Kt 90/k 207, porte en son revers des traces de textile, de ficelles et de fils. Le texte indique qu'il s'agit d'un envoi d'Aššurmalik, fils d'Innaya, scellé par Alulu et le serviteur d'Aššur-malik ${ }^{34}$. Ces personnes ne sont pas attestées non plus dans l'archive de Šumī-abiya. Notons que dans sa description de l'objet, N. Özgüç précise qu'il a été découvert dans la même archive que la bullce Kt 91/k 380. Or cette dernière, qui porte juste la mention du sceau de $[\mathrm{X}-\mathrm{x}]$-idī, fils de Zuzanum, a été incluse par K. R. Veenhof dans l'archive d'Elamma exhumée en 1991 ; l'auteur note que ce personnage n'est pas mentionné dans l'archive en question ${ }^{35}$. Les autres bullae découvertes en

${ }^{30}$ N. Özgüç, In N. Özgüç et Tunca $2001: 220$ à 221 et commentaire p. 134.

${ }^{31} \mathrm{KIŠIB} \mathrm{du}_{10}-A$-šur, na-áš-pár-tum, ša A-šur-i-di, [x x]-ni?.

${ }^{32}$ Kt 90/k 206 est une bullce de forme hémisphéroïde avec un revers concave qui porte les traces d'un nœud et de fils qui en partent. Le texte est le suivant : kišib kà-ri-im Kà-ni-iš, a-šu-mì A-bu-ša-lim dити Ku-ra-ra. Y figurent également deux impressions partielles d'un sceau identique à celui imprimé sur l'enveloppe fragmentaire d'une reconnaissance de dette (Kt 90/k $297=$ TPAK 1, 137).

${ }^{33}$ Kt 90/k 209, [X-X-X]-lí dumu E-di-n[a-a], [X-X-X-X] dumu Ša-lim$A-s ̌[u ̀ r],[\mathrm{X}-\mathrm{X}-\mathrm{X}]-\check{s} u$ kišib ${ }^{\mathrm{i} i m}-[\mathrm{X}-\mathrm{X}-\mathrm{x}],[\mathrm{X}$ x x $] \mathrm{gudu}_{4} e-[\mathrm{X}-\mathrm{x}],[\mathrm{X}-\mathrm{x}]-\check{s} a-$ ma $m a-n u[m \mathrm{x}-\mathrm{x}]$.

${ }^{34} \mathrm{Kt}$ 90/k 207 : kišib A-lu-lu ú șú-ha-ru-i[m], ša A-šùr-ma-lik, na-ášpár ${ }^{a r}$-tum $\check{s} a A$-šùr-/ma-lik, dumu E-lá-li; elle porte deux impressions partielles d'un sceau.

${ }^{35}$ AKT 8 (Veenhof sous presse), texte 368 et p. 465 ; je remercie $\mathrm{K}$. R. Veenhof pour m'avoir communiqué son manuscrit avant publication.
}

1990 ne sont pas inscrites ou encore appartiennent à une autre archive ${ }^{36}$.

\section{Les bullæ dans la maison d'Elamma (1991)}

En 1991, une maison située dans les carrés LVI/127-128 a été dégagée (figure 1) ; les 365 tablettes, enveloppes et les 7 bullae qui y étaient conservés appartiennent aux archives d'Elamma dont l'agencement est décrit par T. Özgüç ci-dessus. K. R. Veenhof, l'éditeur de cette archive, note que, en l'absence d'un rapport de fouilles détaillé, ces informations ne suffisent pas à reconstituer le classement original des tablettes ${ }^{37}$. Parmi les 7 bulloe exhumées avec l'archive, dont 4 inscrites, une seule (Kt 91/k 378) pourrait être liée à une ou plusieurs tablettes de l'archive ; les trois autres bullce inscrites font intervenir des individus non attestés par ailleurs.

\section{Les bullæ dans la maison de Šalim-Aššur (1994)}

En 1994, la maison de Šalim-Aššur et de ses deux fils Ennam-Aššur, et d'Ali-ahum contenait 1100 textes et 33 bullae, dont 19 inscrites. Selon M. T. Larsen qui édite cette archive $^{38}, 7$ bullce auraient servi à identifier des envois de marchandises ou de tablettes et 11 autres auraient eu une fonction archivistique : elles permettaient l'identification de lots de tablettes ${ }^{39}$; la dernière porte un texte trop fragmentaire pour en définir l'usage. L'examen des textes présents sur ces 11 bullce a permis à M. T. Larsen de relier 5 d'entre elles à des lots de tablettes existant dans l'archive, 3 autres proposent des désignations suffisamment large pour concerner un nombre important de tablettes, les 3 dernières n'ont pas de lien apparent avec les tablettes exhumées dans la maison ${ }^{40}$.

\section{Les bullæ dans la maison d'Ali-ahum (1993)}

En 1993, les archéologues ont mis au jour deux maisons voisines dans les carrés LVII/127 et LVIII/127-128, aux contours indéfinis en raison de leur état de destruction avancé ; ces maisons recelaient 926 tablettes et 37 bullae constituant les archives d'Ali-ahum, fils d'Iddin-Suen, et de son fils Aššur-taklāku ${ }^{41}$. Une fois de plus, tablettes et bullae ont été malheureusement inventoriées à part.

Les bullce se répartissent en deux grands groupes :

- 20 bullae dont les numéros d'inventaire sont consécutifs Kt 93/k 254 à Kt 93/k 273 auraient été découverts dans la « maison $1 », \mathrm{LVII} / 127$, au

\footnotetext{
${ }^{36}$ Kt 90/k 49 avec une empreinte de tissus fin ; Kt 90/k 112 de forme triangulaire avec un trou pour passer une ficelle et deux impressions d'un même sceau; Kt 90/k 210 avec deux trous de ficelles et des marques profondes sur le dos, ainsi que l'impression partielle d'un sceau. À partir de Kt 90/k 429, les tablettes appartiennent à une autre archive étudiée par S. Çeçen.

${ }^{37}$ Introduction d'AKT 8.

${ }^{38} \mathrm{AKT} 6 \mathrm{a}$; AKT 6b ; d'autres volumes sont à paraître.

${ }^{39}$ Larsen $2008: 85$.

${ }^{40}$ Larsen $2008: 86-87$.

${ }^{41}$ Michel 2008, 2015 ; N. Özgüç et Tunca 2001 : 229-235, 342-345.
} 


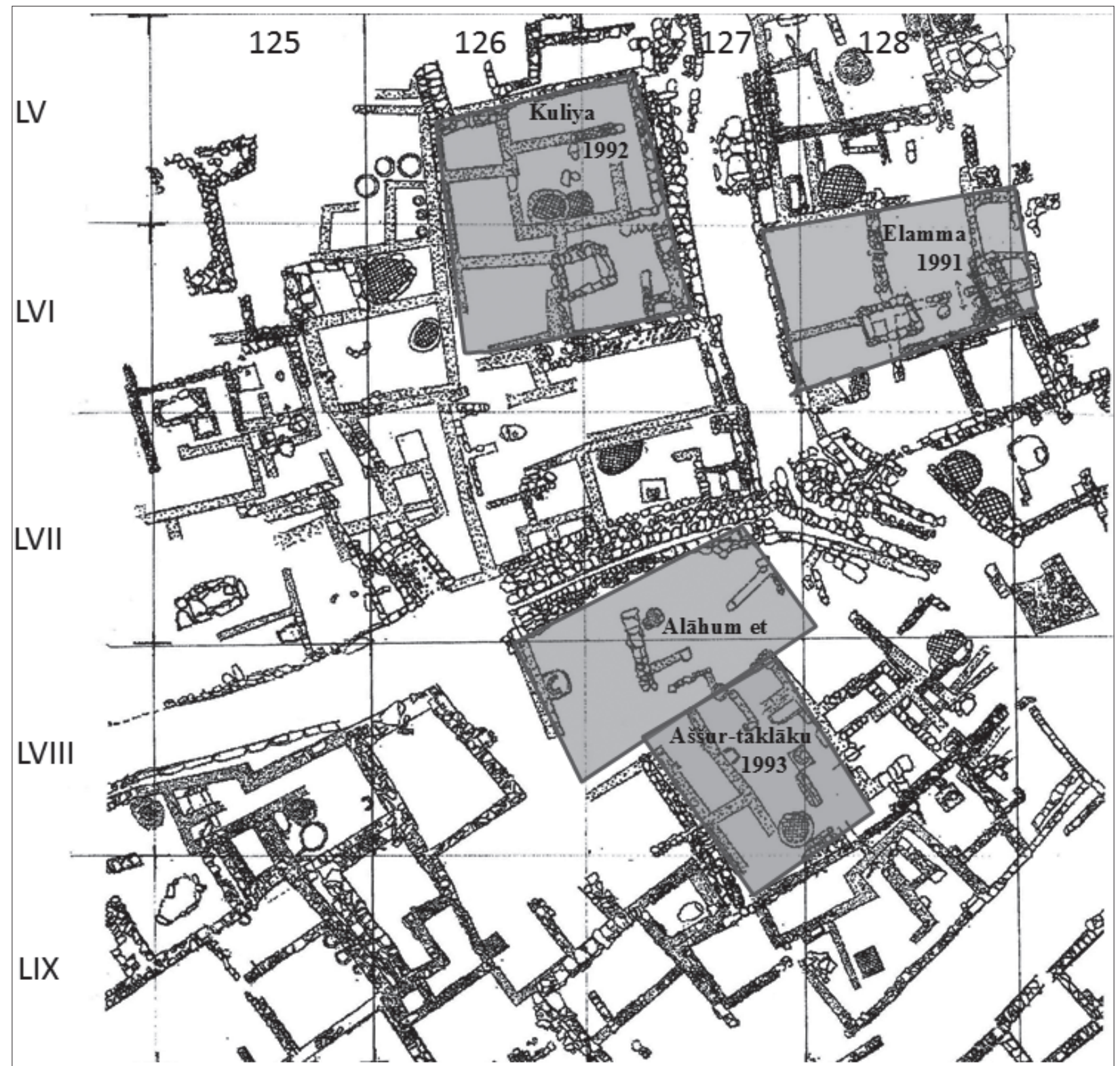

Figure 1 : Secteur de la ville basse exploré entre 1991 et 1993. (Mission ARChÉologiQue de Kültepe).

sud de la rue pavée en pierre. Deux autres bulloe, aux numéros d'inventaire isolés, seraient également issues de cette maison : Kt 93/k 608 et Kt 93/k 650.

- 15 bullae dont les numéros d'inventaire sont consécutifs Kt 93/k 801 à Kt 93/k 815 proviendraient de la «maison 2 », LVIII/127-128.

Vingt de ces bullae sont anépigraphes. Les bullce Kt 93/k 254 à Kt 93/k 257, ainsi que Kt 93/k 608 sont en forme de disque (ou demi-disque) avec une face bombée portant l'empreinte d'un sceau et une face plate traversée par l'empreinte d'une corde ; ils portent tous la même empreinte de sceau dont le propriétaire n'est pas identifié (figure 2a-b). Ces bullce ont sans doute servi à sceller des caisses ou des portes en bois. Il est probable que les bullce Kt 93/k 260 et Kt 93/k 261 avaient à l'origine la même forme et un usage similaire. La bulloe Kt 93/k 258 ressemble à un disque qui aurait été appliqué sur l'arrête d'un coffre ou du bâti dormant d'une porte ; l'empreinte de sceau qu'elle porte est à peine visible. Les bulloe Kt 93/k 259 et Kt 93/k 262 ont été appliquées autour d'une ficelle ou d'une corde et présentent des empreintes de sceaux. La bullce Kt 93/k 650 a été appliquée sur des ficelles qui se croisent perpendiculairement ; on distingue des traces de textile et de fils. La bulloe Kt 93/k 801, en forme de demi-sphère comporte cinq empreintes de sceaux. Sur le revers, on observe l'empreinte très claire d'un panier dont les roseaux sont agencés de manière concentrique (figure 3a-b). Kt 93/k 809 

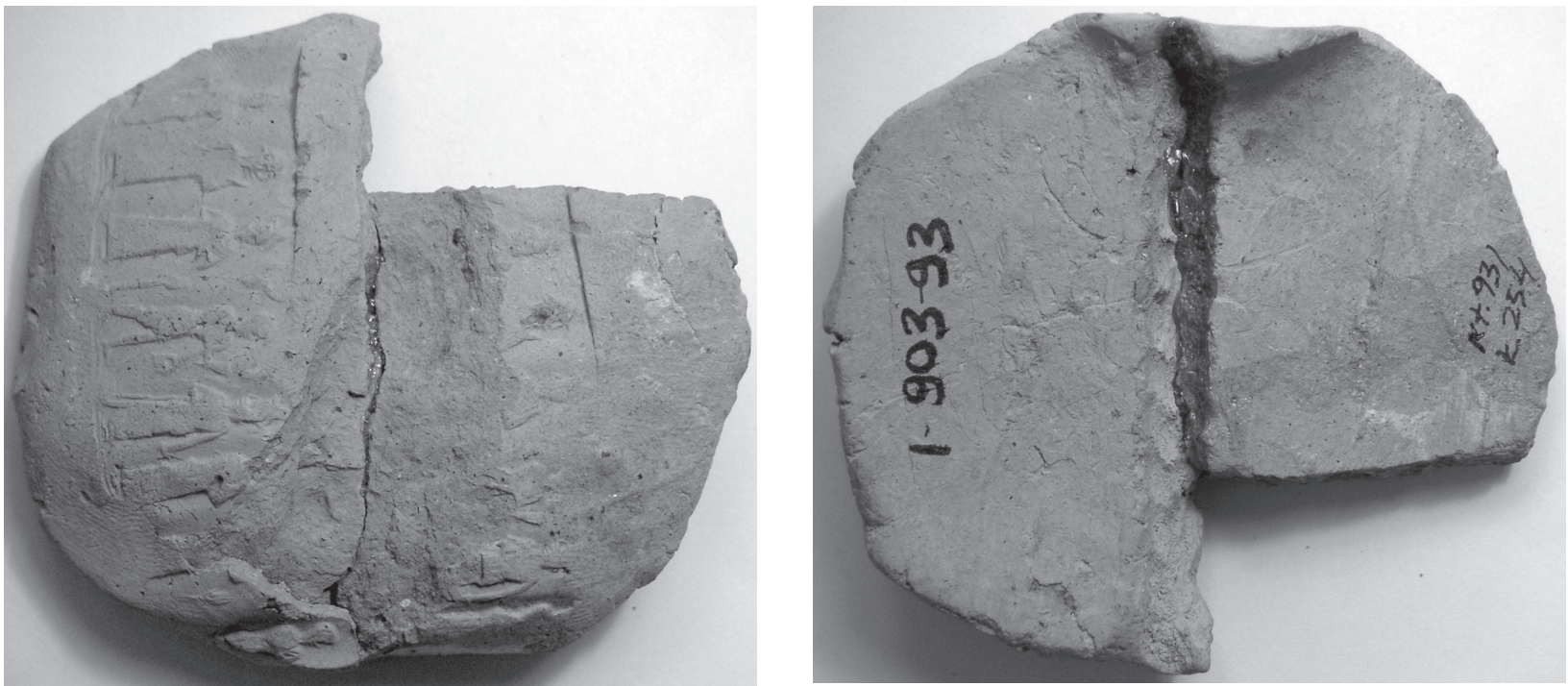

Figure 2 : Bulla Kt 93/K 254 ; A. FACE ; B. ReVers. Photo C. Michel.
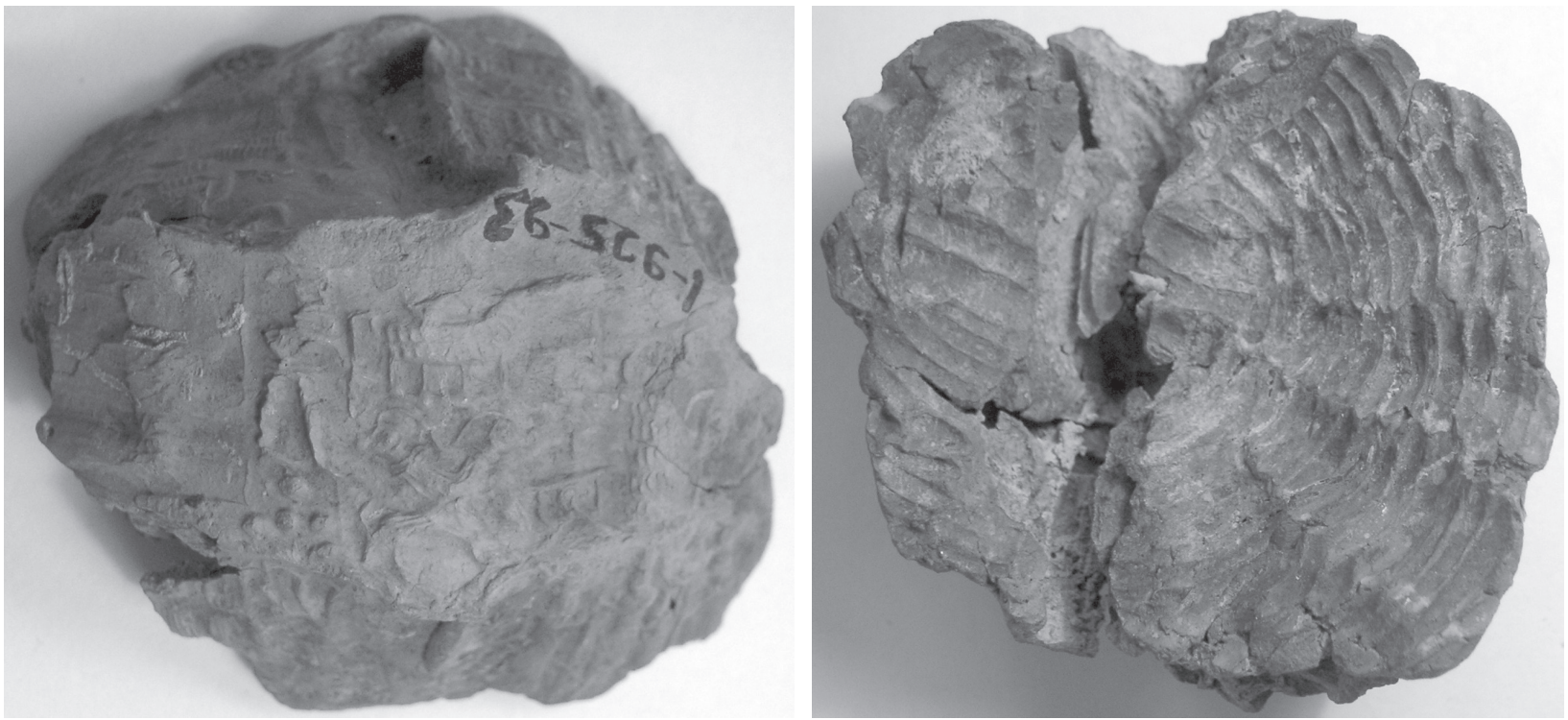

Figure 3 : Bulla Kt 93/K 801 ; A. FACE ; B. Revers. Photo C. Michel.

porte l'impression du sceau d'Aššur-taklāku, l'un des propriétaires de la maison, et au revers montre les marques d'une structure de panier. Kt 93/k 810 a sur une face l'empreinte d'un sceau-cylindre et, sur l'autre, des traces de roseaux et de profondes rainures. Kt 93/k 267 est un bouchon d'argile en forme de champignon avec des impressions de sceau-cylindre et de sceau tampon en forme d'abeille et des marques de ficelles au revers. Les bullce Kt 93/k 266, Kt 93/k 803, Kt 93/k 812, Kt 93/k 814 , Kt $93 / \mathrm{k} 815$ sont trop fragmentaires ; on distingue juste des restes d'empreintes de sceaux. Ces différentes bullae ont donc servi à sceller des portes, des caisses en bois, des paniers, des ballots de marchandises emballées dans du tissu et des récipients à col.
Les 17 autres bullae sont inscrites avec des textes plus ou moins longs. Parmi elles, 7 bullæ concernent des envois, našpertum ; la plupart ne sont pas scellées.

- Kt 93/k 263 mentionne un envoi, sans doute de tablettes, de Kurub-Ištar, scribe de Timilkiya, relatif à Aššur-malik, fils d'Usum, et Pūšu-kēn, fils de Šarawa ${ }^{42}$. Kurub-Ištar, Aššur-malik et Pūšukēn sont bien attestés dans le corpus de tablettes exhumé en 1993, mais les deux derniers figurent avec des patronymes différents ou sans patronyme, et les trois personnages n'apparaissent jamais

\footnotetext{
${ }^{42} \mathrm{Kt}$ 93/k 263, n[a]-ášs-pè-er-tí [ša], Kur-ub-Ištar dumu $A$-[x-x-x], dub-sar ša Tí-mì-i $\left[l_{5}-k i-a\right], a$-šu-mì A-šur-m[a-lik], dumu Ú-sí-im ú $P[u-\check{s} u-k e]-/ e\left[n^{6}\right]$, dumu $\breve{S} a-r a-w a$.
} 

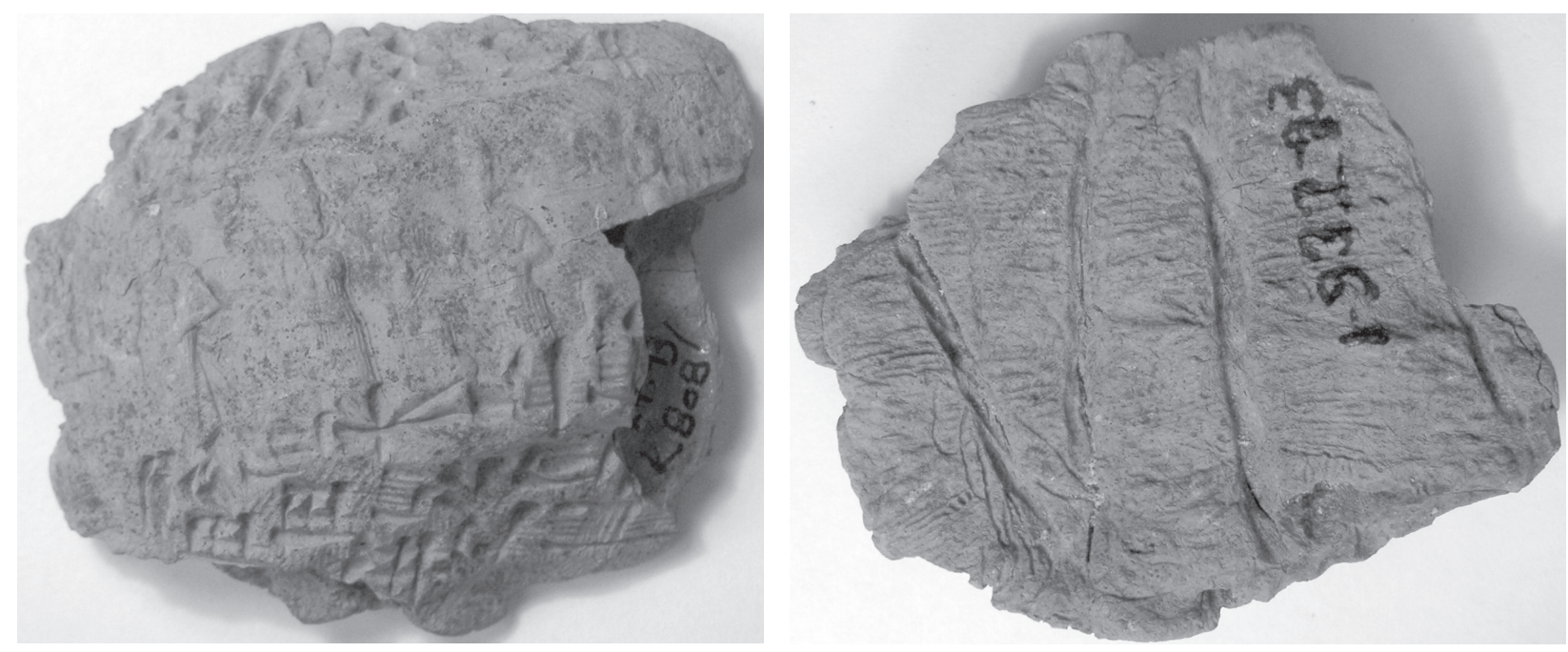

Figure 4 : Bulla Kt 93/K 808 ; A. FACE ; B. ReVers. Photo C. Michel.

ensemble. Kurub-Ištar pourrait éventuellement être le fils d'Aššur-Šamš̄̄ qui intervient comme codestinataire d'une lettre sous enveloppe (Kt 93/k 379:3). Kurub-Ištar apparaît à deux reprises comme expéditeur d'une lettre à IddinSuen (Kt 93/k 251 et son envelope Kt 93/k 134 ; Kt 93/k 253) et une fois comme l'auteur d'une lettre à Ali-ahum (Kt 93/k 193). Le revers plat de cette bullae présente des rayures (bois ?) et est traversé par l'empreinte de deux ficelles qui se croisent perpendiculairement; elle pourrait avoir servi à sceller un petit coffre à tablettes.

- Kt 93/k 264 note un envoi d'Aššur-idīi3. Cet anthroponyme apparaît à maintes reprises dans les archives découvertes en 1993 ; ce texte est trop succinct pour pouvoir établir un lien précis entre cette bullae et une ou plusieurs tablettes. Les traces au revers ne permettent pas de postuler l'usage de cette bullae.

- Kt 93/k 268 enregistre aussi un envoi, mais le nom de l'expéditeur a disparu ${ }^{44}$. Au revers figurent les empreintes parallèles de trois cordes (?).

- Kt 93/k 271 concerne un envoi de Hinnaya à propos des ânes d'Elamma et de Laqēp ${ }^{45}$. Hinnaya est l'associé commercial d'Elamma (frère d'Aliahum) est très présent dans les archives de ce dernier (cf. AKT 8). Dans les archives d'Ali-ahum, il apparaît comme propriétaire de marchandises étoffes et étain - envoyées en Anatolie (Kt 93/k $150 ; 155 ; 183 ; 187 ; 317 ; 763 ; 893)$. Le dos de cette bullce présente une large empreinte d'un roseau plat et des empreintes de textile.

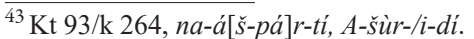

${ }^{44} \mathrm{Kt}$ 93/k 268, [na-á] š-pè-er'-/tí, [X-X-x]-ma?.

${ }^{45} \mathrm{Kt}$ 93/k 271 na-áš-pár-tum, ša Hi-na-a, ša a-šu-mì, anše, ša E-lá-ma, ù Lá-qé-ep.
}

- Kt 93/k 272 mentionne un envoi de Kurub-Ištar qui concerne également Agūa et son fils Ușur-šaAššur ${ }^{46}$. Celui-ci apparaît régulièrement comme témoin dans les transactions impliquant les propriétaires des archives. Au revers, les traces ne sont pas identifiables.

- Kt 93/k 808 consigne un envoi d'Aššur-țāb et porte le sceau de Malahum (ou du batelier) ; il s'agirait d'un envoi d'étain (figure $4 a-b)^{47}$. Les traces de textile et de ficelles torsadées au revers montrent que cette bullce a servi à sceller de la marchandise emballée dans une étoffe, ou encore des tablettes.

- Kt 93/k 804 a été scellé par trois marchands et concerne un envoi, mais le nom de l'expéditeur a disparu ${ }^{48}$. Au revers, on distingue des traces d'étoffes et de ficelles.

Plusieurs bullae mises au jour en 1993 ont été appliquées sur des contenants à tablettes et ont donc servi au classement des archives d'Ali-ahum ou de son fils Aššurtaklāku.

- Kt 93/k 273, scellée par Ali-ahum, fermait un lot de tablettes certifiées, verdicts du kārum à propos de Suen-pilah ${ }^{49}$. L'ensemble était protégé d'un

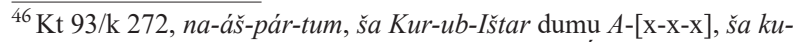
$n u$-ki-šu lá pá-t[i], ša 12 ma-na an-na, ša a-na Ú-șú-ur-ša-[A-šùr], dumu $A-g u_{5}-u$-a ǐ̌s-tù $A-l\left[i m^{\mathrm{ki}} \ldots\right]$, ù Kur-ub-Ištar ki-ma $\check{s} u$-[a-ti], il $I_{5}$ qé-ú-ma ù $i-a-t[i], U_{-s ̦ u} u r-s ̌ a-A$-šùr, lá $i$-tù-ra-ni-[ma], i-pá-té-šu a-dí, [n]i-na-mu-ru.

${ }^{47} \mathrm{Kt} \mathrm{93/k} \mathrm{808,} \mathrm{na-áš-pè-[er-tu]m,} \mathrm{ša} \mathrm{A-šùr-du}{ }_{10}$, kišib Ma-lá-hi-im, (sceau), a-dí-i : an-na $s ̌\left[a \mathrm{x}\right.$ x], lu síg? ša Ma-[a-ma], il ${ }_{5}-q e ́-u ́$.

${ }^{48}$ Kt 93/k 804, (sceau A), [kišib ${ }^{2}$ Puzur - sú-e[n $]$, [kišib] Puzur $-a-n a$,

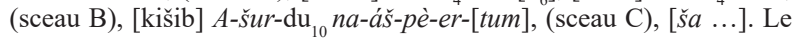
sceau A est inscrit à l'envers : Puzur $_{4}-a-s ̌ s[u ̈ r]$, dumu $E$-la-[li] $=$ Tessier 1994, n²70 ; le sceau C est inscrit: $A$-šur-du ${ }_{10}$ dumu, $A$-lá-hi-im = Tessier 1994, n³59.

${ }^{49} \mathrm{Kt}$ 93/k 273, tup-pu-um, ha-ar-mu-um ša, dí-in kà-ri-im ša, a-šu-mi $S[\dot{u}-e] n_{6}$-pá-lá-ah, (sceau inscrit: $[A-l]$ á-hu-um, dumu $A$-šùr-du $\left.{ }_{10}\right)$.
} 

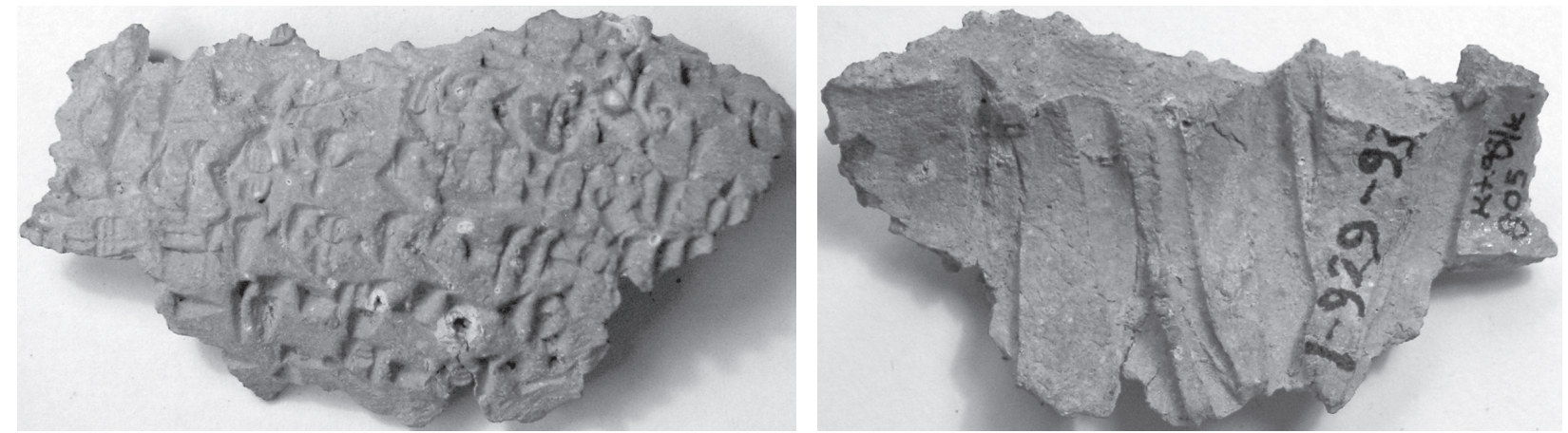

Figure 5 : Bulla Kt 93/K 805 ; A. FACE ; B. ReVers. Photo C. Michel.

tissu fermé par des liens en roseau. Toutefois, les tablettes ainsi emballées n'ont pas pu être identifiées car Suen-pilah est absent de l'index des anthroponymes des tablettes exhumées en 1993.

- Kt 93/k 806 fermait un lot de tablettes certifiées relatives à $[\mathrm{X}] \operatorname{aršu}^{50}$; aucun nom finissant ainsi n'est attesté dans les archives trouvées en 1993.

- Kt 93/k 807 identifiait des tablettes appartenant ou concernant Aššur-damiq ${ }^{51}$; cet anthroponyme est cité à trois reprises comme éponyme dans une date ${ }^{52}$.

- Kt 93/k 813 était sans doute fixée sur un panier comportant des verdicts de l'assemblée d'Aššur et scellés par le kārum de Kanešs ${ }^{53}$.

- Les autres bulla inscrites découvertes en 1993 portent soit des textes assez longs, très proches de ceux que l'on trouve sur les tablettes, soit quelques signes, fragments de mots non identifiables.

- Kt 93/k 270 est une bullae peu épaisse avec un texte de 9 lignes sur la face et un revers qui porte des impressions de sceaux ténues et une empreinte de ficelle. Le texte, adressé à un interlocuteur à la deuxième personne du singulier, concerne des étoffes disponibles à la vente et une somme d'argent emportée par quelqu'un ${ }^{54}$.

- Kt 93/k 805, appliqué sur une structure en roseaux, est un fragment de bullce avec un texte de 7 lignes se terminant par la mention de trois témoins, comme une déposition (figure $5 \mathrm{a}-\mathrm{b})^{55}$. Il n'a pas d'empreinte de sceau.

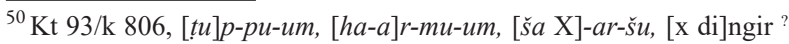
$\mathrm{x},\left[\begin{array}{lll}\mathrm{x} & \mathrm{x} & \mathrm{x}\end{array}\right]$.

${ }^{51} \mathrm{Kt}$ 93/k 807, (sceau), tup-pu-ù, ša A-šùr-/sig !

${ }^{52}$ Kt 93/k 361: 24 ; Kt 93/k 449:16; Kt 93/k 275: 20 (ša qāti A.).

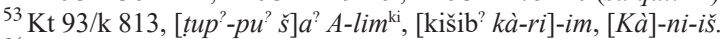

${ }^{54} \mathrm{Kt} \mathrm{93/k} \mathrm{270,} \mathrm{ša} \mathrm{túg-hi-a-kà,} \mathrm{a-ma-lá} \mathrm{té-er-tí-k[à],} \mathrm{i-ba-ší-ú} \mathrm{ù} \mathrm{kù-}$ babbar, qá-at-kà ša dumu!, [Lu]-zi-na a-sí-ib-/tim, [ü?-śé-b]i-lá-ma kù-babbar-ma, [ša a-h]u-kà, [i-lá-q]é-ú, [a-na túg]-hi-a-k[à].

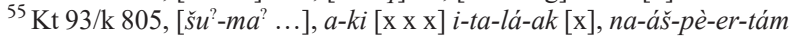

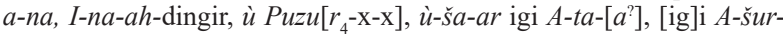
sig $_{5}$, [ig]i En-na-nim.
}

- Kt 93/k 269 comporte trois lignes de texte avec deux impressions d'un sceau inscrit au nom d'Aššur-taklāku, fils d'Ali-ahum. Le texte, très fragmentaire, fait référence à des offrandes votives $^{56}$. Le revers est marqué par des empreintes de roseaux et ficelle.

- Kt 93/k 802 porte la mention de deux sceaux et deux empreintes de sceaux ${ }^{57}$. Les marques au revers sont indéfinissables.

- Kt 93/k 265 présente sur son revers des traces de roseaux parallèles; sur la face ne subsiste que la mention du sceau de Šum[X-X-X] et l'impression fragmentaire de celui-ci ${ }^{58}$.

- Kt 93/k 811 est un fragment sur lequel ne subsiste que quelques signes. Le revers porte des marques de fils, ficelles et roseaux ${ }^{59}$.

Une petite moitié des bulla inscrites exhumées en 1993 a donc servi à sceller des ballots de marchandises ou des tablettes expédiées souvent depuis Aššur, un quart a permis d'identifier des lots thématiques de tablettes, essentiellement des textes à caractère juridique, l'usage du dernier quart n'a pas pu être défini. Notons enfin qu'une dizaine des bullce découvertes en 1993 présentent des empreintes digitales claires ${ }^{60}$, qui pourraient éventuellement permettre une dactyloscopie.

L'étude des bullae de Kültepe, en l'absence de repères archéologiques, se concentre nécessairement sur les objets eux-mêmes. La forme générale, ainsi que les traces ou marques laissées sur l'envers de ces morceaux d'argile, témoignent parfois de leur usage : une surface

\footnotetext{
${ }^{56} \mathrm{Kt} \mathrm{93/k} \mathrm{269,} \mathrm{(sceau),} \mathrm{[...]} \mathrm{ša} \mathrm{ik-ri-bi}{ }_{4}-[\mathrm{x}]$, [...] ma-ma-an, (même sceau), [x x] x-té-šli ?.

${ }^{57} \mathrm{Kt} 93 / \mathrm{k} 802$, (sceau A imprimé à l'envers), [kišib $X$-n] $a-A$-šur kišib Lu-zi-[(x)], (sceau B). Les noms Luzi, Luzia et Luzina sont attestés dans l'archive de 1993.

${ }^{58}$ Kt 93/k 265, kišib $\breve{S} u-m[x-x-x-x]$, [dumu X-x]-ma?

${ }^{59} \mathrm{Kt}$ 93/k 811, (seau), [...]-da- $a$, [...]-tù- $a a^{?}$.

${ }^{60}$ Kt 93/k 256, 257, 260, 264, 508, 803, 809, 810 et 811 .
} 
plate suggère par exemple l'application sur une porte ou une caisse en bois, des traces de cordages, de roseaux et de textiles font penser à la fermeture d'un ballot ou sac en tissu. Les bullae anépigraphes portent systématiquement l'empreinte d'un sceau permettant d'identifier l'auteur du scellement. Les bullce inscrites, quant à elles, ont étiqueté contenants et ballots renfermant marchandises et tablettes. Dans les archives, les bullce ont servi à la fois de scellements et d'étiquettes pour identifier des textes classés ensemble. En raison d'un inventaire des objets inscrits, non pas en fonction de leur provenance précise mais selon leur forme ou leur usage, le lien entre une bullce inscrite et les tablettes auxquelles elle se réfère est perdu. L'inscription sur les bulla montrent qu'elles ont servi au classement des tablettes par dossier ou par genre ; les textes à caractère juridique - verdicts, tablettes certifiées, créances - semblent avoir été systématiquement classés à part au sein d'une archive. Plus l'archive est importante, plus il devrait y avoir de bullae; cela est en effet le cas pour les archives découvertes en 1993 et 1994.

\section{Abréviations}

AKT 4 İ. Albayrak, (Ankara) Kültepe Tabletleri (Kt o/k) 4, Türk Tarih Kurumu Yayınları VI/33b, Ankara, 2006

AKT 6a M. T. Larsen, (Ankara) Kültepe Tabletleri VIa. The Archive of Šalim-Aššur, Family volume 1, The First Two Generations, Türk Tarih Kurumu Yayınları VI/33c, Ankara, 2010.

AKT 6b M. T. Larsen, (Ankara) Kültepe Tabletleri VI-b, The Archive of Šalim-Aššur, Family volume 2, Ennam-Aššur, Türk Tarih Kurumu Yayınları VI/33d-b, Ankara, 2013.

AKT 8 K. R. Veenhof, (Ankara) Kültepe Tabletleri VIII, The Archive of Elamma, son of IddinSuen, and his Family (Kt 91/k 285-568 and Kt 92/k 94-187), Türk Tarih Kurumu Yayınları VI/33f, Ankara (sous presse).

TPAK 1 C. Michel et P. Garelli, Tablettes paléoassyriennes de Kültepe 1 (Kt 90/k), Paris, Istanbul, diff. De Boccard, 1997.

\section{Bibliographie}

Albayrak, İ. 1996. Koloni çaginda yerli bir bayan Madawada, III. Uluslararasi Hititoloji Kongresi Bildirileri, Corum 16-22 eylül 1996 : 114.

- 2000. Ein neues altassyrisches Testament aus Kültepe. Archivum Anatolicum 4 : 1-16 (turc) : 17-27 (allemand).

— 2001. Kültepe metinlerinde geçen ma'u 'su' kelimesi. Anadolu Medeniyetleri Müzesi 2000 yıllı̆̆g, Ankara : 300-311.
- 2005a. Kumaş ve Kalay Sevkıyatına İlişkin üç Kültepe Tableti. Anadolu Medeniyetleri Müzesi 2003-2004 yıllığı Ankara : 269-277.

- 2005b. 1963 Kazılarında Ele Geçen Yerli Halkla Ilgili Bir Grup Kültepe Tableti. 5.UHKB Çorum 2002, Ankara : 21-32.

- 2006. Ana kaspim annīm Šamaš-abì qātātum 'Bu gümüş için Šamaš-abī kefildir'. (ana kaspim annīm Šamaš-ab̄ qàtātum 'Šamaš-abī is guarantor for this Silver'), Archivum Anatolicum 7/2 : 1-12.

Alp, S. 1968. Zylinder-und Stempelsiegel aus Karahöyük bei Konya, Ankara, Türk tarih kurumu basimevi.

Erol, H. et Albayrak, İ. 2012. Kültepe'den nikka İlgili Alt1 Yeni Belge. Archivum Anatolicum 8/2 : 1-20.

Ezer, S. 2014. Kültepe-Kanesh in the Early Bronze Age. In L. Atici, F. Kulakoğlu, G. Barjamovic et A. Fairbairn (éd.), Current Research at Kültepe-Kanesh. An Interdisciplinary and Integrative Approach to Trade Networks, Internationalism, and Identity. Journal of Cuneiform Studies, Supplemental Series 4 : 5-24. Atlanta, Lockwood Press.

Frangipane, M., Ferioli, P., Fiandra, E., Laurito, R., et Pittman, H. 2007. Arslantepe Cretulae: An Early Centralised Administrative System Before Writing. Roma, Università di Roma La Sapienza.

Hertel, T. 2013. Old Assyrian Legal Practices. Law and Dispute in the Ancient Near East. Old Assyrian Archives, Studies 6, Publications de l'Institut historique-archéologique néerlandais de Stamboul 123. Leiden, Nederlands Instituut voor het Nabije Oosten.

Kulakoğlu, F. 2015. Current Researches at KültepeKanesh. In F. Kulakoğlu et C. Michel (éd.), Proceedings of the 1st Kültepe International Meeting, Kültepe, September 19-23, 2013 (Kültepe International Meetings 1, Subartu XXXV), Turnhout, p. 9-21.

Larsen, M. T. 1977. Seal Use in the Old Assyrian Period. In Mc. G. Gibson, et R. D. Biggs (éd.), Seals and Sealing in the Ancient Near East, Bibliotheca Mesopotamica 6, Malibu, Undena Publications : 89105.

— 2002. The Aššur-nādā Archive, Old Assyrian Archives 1. Publications de l'Institut historique-archéologique néerlandais de Stamboul 96. Leiden, Nederlands Instituut voor het Nabije Oosten.

- 2008. Archives and filing systems at Kültepe. In C. Michel (éd.), Old Assyrian Studies in Memory of Paul Garelli. Old Assyrian Archives Studies 4. Publications de l'Institut historique-archéologique néerlandais de Stamboul 112 : 77-88. Leiden, Nederlands Instituut voor het Nabije Oosten.

Michel, C. 1994. Règlement des comptes du défunt Huraşānum. Revue d'Assyriologie et d'Archéologie Orientale 88 : 121-128 (http://halshs.archivesouvertes.fr/halshs-00821254).

- 2000. A propos d'un testament paléo-assyrien: une femme 'père et mère' des capitaux. Revue d'Assyriologie et d'Archéologie orientale 94 : 1-10 
(http://halshs.archives-ouvertes.fr/halshs-00642823/ $\mathrm{fr} /)$.

- 2001. Correspondance des marchands de Kaniš au début du II e millénaire av. J.-C.. Littératures du Proche-Orient ancien 19. Paris, Éditions du Cerf.

- 2003. Old Assyrian Bibliography of Cuneiform Texts, Bullce Seals and the Results of the Excavations at Assur, Kültepe/Kanis, Acemhöyük, Alishar and Bogazköy. Old Assyrian Archives Studies 1. Publications de l'Institut historique-archéologique néerlandais de Stamboul 97. Leiden, Nederlands Instituut voor het Nabije Oosten.

- 2006. Old Assyrian Bibliography 1 (February 2003 - July 2006). Archiv für Orientforschung 51 (20052006) : 436-449.

- 2008. The Alāhum and Aššur-taklāku archives found in 1993 at Kültepe Kaniš. In G. Kryszat (éd.), Festschrift für Karl Hecker zum 75. Geburtstag am 25. Juli 2008, Altorientalische Forschungen 35: 5367 [résumé Altorientalische Forschungen 35: 359] (http://halshs.archives-ouvertes.fr/halshs-01186428).

- 2009. Les femmes et l'écrit dans les archives paléo-assyriennes $\left(\mathrm{XIX}^{\mathrm{e}}\right.$ s. av. J.-C.). In F. BriquelChatonnet, S. Fares, B. Lion et C. Michel (éd.), Femmes, cultures et sociétés dans les civilisations méditerranéennes et proches-orientales de l'Antiquité, Topoi Suppl. 10, Lyon: 253-272 (http:// halshs.archives-ouvertes.fr/halshs-00644211/fr/).

- 2011a. Old Assyrian Bibliography 2 (August 2006 April 2009). Archiv für Orientforschung 52 (20072010) : 416-437.

- 2011b. The Private Archives from Kaniš Belonging to Anatolians. In M. Balza (éd.), Archival, Scribal, and Administrative Spaces among the Hittites, Altorientalische Forschungen 38 : 94-115.

- 2015. Women in the Family of Ali-ahum son of Iddin-Suen (1993 Kültepe archive). In F. Kulakoğlu and C. Michel (éd.), Proceedings of the 1st Kültepe International Meeting, Kültepe, 19-23 September, 2013. Studies Dedicated to Kutlu Emre, Kültepe International Meetings 1 : 85-93. Subartu XXXV. Turnhout, Brepols.
Öz, E. et Albayrak, İ. 2012. 1963 Yı1ı Kültepe Kazısında Ele Geçen Altı Yeni Tablet. Archivum Anatolicum $8 / 2: 21-45$.

Özgüç, N. et Tunca, Ö. 2001, Kültepe-Kanis. Sealed and Inscribed Clay Bullce, TTKY V. Dizi - Sayi 48, Ankara.

Özgüç, T. 1994. A Boat-Shaped Cult-Vessel from the Karum of Kanish. In H. Gasche, M. Tanret, C. Janssen et A. Degraeve (éd.), Cinquante-deux réflexions sur le Proche-Orient ancien offertes en hommage à Léon de Meyer : 369-375. Leuven, Peeters.

Tessier, B. 1994. Sealing and Seals on Texts from Kültepe kārum Level 2, Publications de l'Institut historiquearchéologique néerlandais de Stamboul 70. Istanbul, Nederlands historisch-archaeologisch Instituut.

Veenhof, K. R. 1993. On the Identification and Implications of Some Bullce from Acemhöyük and Kültepe. In M. J. Mellink, E. Porada et T. Özgüç (éd.), Aspects of Art and Iconography: Anatolia and its Neighbors. Studies in Honour of Nimet Özgüç, Ankara, Türk tarih kurumu basimevi : 645657.

- 2003. Archives of Old Assyrian Traders. In Brosius, M. (éd.), Archives and Archival Tradition. Concepts of Record-Keeping in the Ancient World: 78-123. Oxford, Oxford University Press.

- 2009. Review of AKT 4. Jaarberitcht van het Vooraziatisch-Egyptisch Genootschap Ex Oriente Lux 41 : 179-202.

- 2011. Last Wills and Inheritance of Old Assyrian Traders with Four Records from the Archive of Elamma. In K. Abraham et J. Fleishma (éd.), Family Cohesion in the Bible and the Ancient Near East. Studies in Honour of Aaron Skaist on the Occasion of his 76th Birthday : 169-201. Bethesda, CDL Press.

- 2013. The Archives of Old Assyrian Traders: their Nature, Functions and Use. In Faraguna, M. (éd.), Archives and archival documents in ancient societies: Legal documents in ancient societies IV, Trieste 30 September-1 October 2011 : 27-71. Trieste, Edizioni Università di Trieste. 